\title{
Clinical Course and Results of Surgery for Chronic Subdural Hematomas in Patients on Drugs Affecting Hemostasis
}

\author{
Tomasz Andrzej Dziedzic, M.D., Ph.D., Przemystaw Kunert, M.D., Ph.D., Andrzej Marchel, M.D., Ph.D. \\ Department of Neurosurgery, Medical University of Warsaw, Warsaw, Poland
}

Objective : An apparent increase of use of drugs affecting hemostasis in our neurosurgical department since the 1990s has encouraged us to investigate whether these drugs influence the clinical course and results of surgery for chronic subdural hematoma (CSDH).

Methods : This retrospective analysis included 178 patients admitted for CSDH from 2007 to 2011 who were divided into two groups : on drugs affecting hemostasis $(40 ; 22 \%)$ and no bleeding disorders (138; $78 \%)$. Medications in the first group included oral anticoagulants $(33 ; 82.5 \%)$, antiplatelets $(5 ; 12.5 \%)$ and low molecular weight heparins $(2 ; 5 \%)$.

Results : The patients on drugs affecting hemostasis were older ( $74.3 \pm 7.4$ vs. $68.4 \pm 14.8 ; p$-value 0.01$)$ and the group without bleeding disorders had more head trauma history (61\% vs. $38 \%, p$-value 0.01$)$. The groups did not differ in bilateral hematoma rates ( $25 \%$ vs. $20 \%, p$-value=NS). At diagnosis, mean hematoma thickness was lower in patients on drugs affecting hemostasis $(18.7 \pm 7.4$ $\mathrm{mm}$ vs. $21.9 \pm 7.9 \mathrm{~mm}, p$-value $<0.01)$. Average stay of hospital was 1 day longer in patients on drugs affecting hemostasis $(11.7 \pm 4.1$ vs.10.9 $\pm 5.3, p$-value $=\mathrm{NS}$ ) and was related to the necessity of bleeding disorder reversal. Mean neurological status at presentation was similar between the groups ( $p$-value=NS) as was the likelihood of hematoma recurrence ( $p$-value=NS). Glasgow Outcome Scale results were comparable.

Conclusion : Patients on drugs affecting hemostasis are less often aware of a head trauma history, possibly suggesting a higher CSDH risk after minor trauma in this group. In these patients, smaller hematomas are symptomatic, probably due to faster hematoma formation. Drugs affecting hemostasis do not affect treatment results.

Key Words : Chronic subdural hematoma · Bleeding disorders · Anticoagulation $\cdot$ The elderly $\cdot$ Trauma.

\section{INTRODUCTION}

The incidence of chronic subdural hematoma (CSDH) related to drugs affecting hemostasis has increased up to $14 \%$ in recent years ${ }^{11,19}$. At the end of the 20th century those patients accounted only for $1.2 \%$ of all patients with $\mathrm{CSDH}^{15}$. The first analysis of outcomes in those patients was performed by Bret et al. ${ }^{3)}$ in 1976. There are some retrospective papers which discuss the differences in outcome between patients on and off anticoagulation or on antiplatelet therapy ${ }^{5,16,22}$. However, none

-Received : December 16, 2015 •Revised : July 11, 2016 •Accepted : July 28, 2016

- Address for reprints : Przemysław Kunert, M.D., Ph.D.

Department of Neurosurgery, Medical University of Warsaw, Banacha 1a 02-097 Warszawa, Poland

Tel : +48-22-599-2575, Fax : +48-22-599-1574, E-mail : pkunert@wp.pl

This is an Open Access article distributed under the terms of the Creative Commons Attribution Non-Commercial License (http://creativecommons.org/licenses/by-nc/4.0) which permits unrestricted non-commercial use, distribution, and reproduction in any medium, provided the original work is properly cited. 
of these papers present data about all kinds of patients with drug affected hemostasis that we meet in neurosurgical practice. A steady increase of patients who are on drugs affecting hemostasis in our department has encouraged us to carry out a retrospective analysis of the results of treatment in patients with this common neurosurgical disorder.

\section{MATERIALS AND METHODS}

A retrospective analysis was performed of 178 patients who were admitted to our department because of CSDH between 2007 and 2011. Patients with small hematomas who did not require surgical treatment were excluded from the analysis. Those patients are not admitted to neurosurgical department. Bleeding disorder which was not related to medication was also an exclusion criteria from the study. Epidemiological data were collected from medical files. Neurological condition on admission and at discharge was based on the Glasgow Coma Scale and physical examination was based on medical files. Information about hematoma characteristics came from available radiological data. This group of patients consisted of $68.6 \%$ (132) men and 31.4\% (56) women. Their ages ranged from 20 to 99 (mean age : 69.4). In all cases diagnosis was based on computed tomography (CT) and confirmed on surgery.

The patients were divided into two groups : one contains patients on drugs affecting hemostasis $(40 ; 22 \%)$ and second group contains patients without bleeding disorder (138; 78\%). All patients without bleeding disorders had a normal clotting and their platelet count was also normal. The patients on drugs affecting hemostasis group were then separated into three subgroups : an oral anticoagulation drugs (OAD) group (33; 82.5\%), an antiplatelet agents (APA) group $(5 ; 12.5 \%)$ and a low molecular weight heparin (LMWH) group (2; 5\%). In 25 $(62.5 \%)$ cases, patients were on medication because of atrial fibrillation, in $6(15 \%)$ cases because of a prosthetic heart valve, in $3(7.5 \%)$ cases because of a coronary stent, in 2 cases because of pulmonary embolism and in 1 case for a heart defect, a stroke, and abdominal aortic aneurysm, respectively. In one case there were no data for the reason for taking medicine. International normalized ratio (INR) of OAD subgroup was above normal range (>1.3). On admission, INR was within the therapeutic range (INR : 2.0-3.0) in 10 (28\%) patients, above the range (INR >3.0) in $12(38 \%)$ patients and under the range
(INR <2.0) in $11(34 \%)$ patients but it exceeded the normal range (1.0-1.3). In patients on APA or LMWH no laboratory abnormalities were identified. The Platelet Function Analysis and the anti-factor Xa assay can be used for monitoring patients on APA and LMWH, respectively. At the time when presented patients were treated these testes were not available on a regular basis in our department.

All patients underwent operation. Burr hole trephination with or without temporary subdural drainage was a standard procedure. Subdural drainage was placed if no significant brain decompression was noted. In cases of multiple septa between internal and external capsules visible on CT scan (13 cases in this group of patients), craniotomy was performed.

In the group on drugs affecting hemostasis, drug discontinuation and equalization of coagulation disorders were undertaken. The speed of reversal of anticoagulation or antiplatelet agent was based on clinical presentation of the patients. In patients who were on anticoagulation, Fresh Frozen Plasma (FFP) or Prothrombin Complex Concentrate (PCC) were used. After identifying reversal of medication a surgery was performed. In all patients on antiplatelet medication at the time of diagnosis drug admission was stopped. When the patients' neurological condition was stable she/he was operated on after seven days. In patients with poor neurological condition platelet concentrate was prescribed and they were operated on immediately. FFP in case of OAD or platelet concentrate in patients receiving antiplatelet medication was delivered. After surgery, LMWH in a prophylaxis dose was prescribed from the first postoperative day. After one week the patients restarts to take OAD and they are on two medications till therapeutic levels of INR are achieved. In the patients who required an antiplatelet medication in perioperative period, acetylsalicylic acid (ASA) was prescribed with reversal of proper treatment after cardiological consultation.

Long-term neurological outcome was assessed at medical appointments in the outpatient clinic with follow-up ranging from 1 to 6 months (mean : 3 months). The results were assessed by means of the Glasgow Outcome Scale.

Data were statistically analyzed with chi-square tests, Fisher's exact tests and Student's t-tests. 


\section{RESULTS}

The group of patients on drugs affecting hemostasis consisted of $40 \%$ (16) women and 60\% (24) men. In the group without bleeding disorders 28.9\% (41) were women and 71.1\% (97) were men; these differences were not significant $(p$-value $=\mathrm{NS})$. In the OAD, the APA and the LMWH groups women represented 42\% (14), 20\% (1) and 50\% (1), respectively. Differences between the subgroups and the group without bleeding disorders were not significant ( $p$-value $=\mathrm{NS}$ ). Patients in the group on drugs affecting hemostasis were older (74.3 \pm 7.4 vs. $68.4 \pm$ 14.8 in the NBD group, $p=0.01$ ) (Table 1). Patients in the OAD subgroup were the youngest (73.2) as compared to those who were on LMWH (78) or APA (80.2). Symptoms, neurological status on admission and history of head trauma are presented in Table 2.
Lateralization of the hematoma was as follows : the group on drugs affecting hemostasis--right 14 (35\%), left 18 (45\%); the group without bleeding disorders-right 46 (34\%), left 57 $(41 \%)$ ( $p$-value=Table 3$)$. A total of $35(25 \%)$ patients had bilateral hematomas in the group without bleeding disorders and 8 (20\%) had bilateral hematomas in the group on drugs affecting hemostasis $(p$-value $=\mathrm{NS}$, chi-square test). None of the patients in the LMWH subgroup had bilateral hematomas. In the APA subgroup the rate of bilateral hematoma was 40\% (2 patients) and in the OAD subgroup 19\% (6 patients). There were no differences between those three subgroups comparing to the group without bleeding disorders. Mean hematoma thickness was statistically significantly lower in the group on drugs affecting hemostasis $(18.7 \pm 7.4 \mathrm{~mm}$ vs. $21.9 \pm 7.9 \mathrm{~mm}$ in the group without bleeding disorders; $p$-value 0.01 , Student's ttest). Mean thickness of subdural hematoma was $19.8 \pm 6.8 \mathrm{~mm}$

Table 1. Epidemiological data

\begin{tabular}{|c|c|c|c|}
\hline Patients & Patients on drug affecting hemostasis $(n=40)$ & Control group $(n=138)$ & $p$-value \\
\hline Female & $16(40)$ & $41(30)$ & NS \\
\hline Male & $24(60)$ & $97(70)$ & \\
\hline Age (years) & $74.3 \pm 7.4$ & $68.4 \pm 14.8$ & 0.01 \\
\hline
\end{tabular}

Values are presented as number (\%) or mean \pm standard deviation. NS : not statistically significant

Table 2. Symptoms, neurological status on admission and history of head trauma

\begin{tabular}{|c|c|c|c|}
\hline Symptoms and signs & Patients on drug affecting hemostasis $(n=40)$ & Control group $(n=138)$ & $p$-value \\
\hline Headache as main complaint & $13(32.5)$ & $37(26)$ & NS \\
\hline GCS on admission & & & NS \\
\hline Min & 7 & 7 & \\
\hline Max & 15 & 15 & \\
\hline Mean & $13.8 \pm 1.8$ & $14.1 \pm 1.4$ & \\
\hline GCS $<15$ on admission & $22(55)$ & $68(49)$ & NS \\
\hline Focal neurological defficit & $18(45)$ & $79(57)$ & NS \\
\hline History of head trauma & $15(39)$ & $84(61)$ & 0.008 \\
\hline
\end{tabular}

Values are presented as number (\%) or mean \pm standard deviation. GCS : Glasgow coma scale, NS : not statistically significant

Table 3. Hematoma lateralization

\begin{tabular}{lccc}
\hline & Patients on drug affecting hemostasis $(\mathbf{n}=\mathbf{4 0})$ & Control group $(\mathbf{n}=\mathbf{1 3 8})$ & p-value \\
\hline Leftsided hematoma & $18(45)$ & $57(41)$ & NS \\
Rightsided hematoma & $14(35)$ & $46(34)$ & NS \\
Bilateral hematoma & $08(20)$ & $35(25)$ & NS \\
\hline
\end{tabular}

Values are presented as number (\%). NS : not statistically significant 
in the OAD subgroup, $18 \pm 5.8 \mathrm{~mm}$ in the APA subgroup and $18.5 \pm 9.1 \mathrm{~mm}$ in the LMWH subgroup, respectively. Statistical difference in terms of mean hematoma thickness was observed $(p$-value $=0.03)$ only between the OAD subgroup and the group without bleeding disorders.

Mean hospital stay was 1 day longer in the group on drugs affecting hemostasis $(11.7 \pm 4.1)$ as compared to the group without bleeding disorders (10.9 \pm 5.3$)$ ( $p$-value=NS, Student's t-test). This difference was related to the necessity for bleeding disorder reversion in the preoperative period. Differences between mean hospital stay were not statistically significant also between the subgroups within the group on drugs affecting hemostasis and the group of patients without bleeding disorders (OAD 11.6 $\pm 4.3 ; p=0.491$, LMWH 9 $\pm 0 ; p$-value $=\mathrm{NS}$, and APA $13 \pm 2.8 ; p$-value $=$ NS). During this time, neurological condition deteriorated in $2(5 \%)$ patients. However, in our group there were no complications related to temporary discontinuation of medication.

Frontal and parietal burr hole trephination with temporary subdural drain placement was the most frequently chosen procedure; 109 (79\%) cases in the group without bleeding dis- orders and $29(72.5 \%)$ in the group on drugs affecting hemostasis (Table 4).

There was no difference in fluid administration in postoperative care between the groups. Mean time of intravenous fluid administration was 5.4 days in the group without bleeding disorders and 5 days in the group on drugs affecting hemostasis ( $p$-value $=$ NS, Student's t-test). Patients with highdose fluid administration (12) of at least $2000 \mathrm{~mL} /$ day for at least 3 days accounted for $60 \%$ (24 patients) in the group without bleeding disorders and $58.6 \%$ (81 patients) in the group on drugs affecting hemostasis $(p$-value $=$ NS, Student's ttest).

The risk of recurrent hematoma (hematoma that required reoperation) was similar between the groups 12 (9\%) patients in the group without bleeding disorders vs. 3 (7.5\%) in the group on drugs affecting hemostasis; ( $p$-value $=$ NS, Fisher's exact test). All reoperated patients from the group on drugs affecting hemostasis were in the OAD subgroup. When only the OAD subgroup was compared with the group without bleeding disorders, the risk of recurrence did not reach a statistically significant difference $(p$-value $=\mathrm{NS})$, either. In all cas-

Table 4. Surgical procedure

\begin{tabular}{|c|c|c|c|}
\hline Procedure & Patients on drug affecting hemostasis $(n=40)$ & Control group $(n=138)$ & $p$-value \\
\hline Burr-hole+drainage & $29(72.5)$ & $109(79)$ & NS \\
\hline Burr-hole & $8(20)$ & $19(14)$ & \\
\hline Craniotomy & $3(7.5)$ & $10(7)$ & \\
\hline \multicolumn{4}{|c|}{ The number of burr-holes } \\
\hline One burr-hole & $3(8)$ & $13(10)$ & NS \\
\hline Two burr-holes & $34(92)$ & $115(90)$ & \\
\hline \multicolumn{4}{|l|}{ Reoperation } \\
\hline Yes & $3(7.5)$ & $12(8)$ & NS \\
\hline No & $37(92.5)$ & $126(92)$ & \\
\hline
\end{tabular}

Values are presented as number (\%). NS : not statistically significant

Table 5. Treatment results in Glasgow Outcome Scale

\begin{tabular}{|c|c|c|c|c|}
\hline Results & Patients on drug affecting hemostasis $(n=40)$ & Control group $(n=138)$ & Total & $p$-value \\
\hline Good recovery & $34(82.5)$ & $123(88)$ & 157/178 (88) & NS \\
\hline Moderate disability & $6(17.5)$ & $11(9)$ & $17 / 178(9)$ & \\
\hline Severe disability & $0(0)$ & $2(1.5)$ & 2/178 (1.5) & \\
\hline Death & $0(0)$ & $2(1.5)$ & $2 / 178(1.5)$ & \\
\hline
\end{tabular}

Values are presented as number (\%). NS : not statistically significant 
es, patients were reoperated during the first hospitalization. In all those cases the previous anticoagulant/antiplatelet therapy was restarted in the way that has been described before.

A total of $82 \%$ patients in the group on drugs affecting hemostasis and 85\% in the group without bleeding disorders were discharged from the hospital after one week. According to the GOS, long-term results of treatment were good and did not differ between the groups ( $p$-value $=$ NS, Fisher's exact test) (Table 5). All patients whose neurological condition was assessed to be a Moderate Disability (6 patients) were in the OAD subgroup ( $p$-value=NS). Two patients died in the group without bleeding disorders (a 75-year-old man with neoplastic disease, due to multi-organ failure; an 83-year-old man, due to cardiac arrest secondary to cardiac arrhythmias). There were no deaths in the group on drugs affecting hemostasis (Table 5). No adverse effects from stopping anticoagulant medication, such as strokes or thromboembolic events were noted in the long-term observation.

\section{DISCUSSION}

Current epidemiological data do not differ from what other groups have reported. CSDHs affect mainly elderly patients, whose mean age is $>65$ years ${ }^{11}$, reaching the highest incidence in the 8th decade of life ${ }^{1,17)}$ and are more common in men ${ }^{13,18)}$. The risk of $\mathrm{CSDH}$ in this group is related to brain atrophy and, possibly, to greater brain movement with an increased tendency to falls ${ }^{2,18)}$. Bridging veins, which are the main source of bleeding, are more prone to tearing in this group ${ }^{25)}$. The trauma is usually trivial and patients do not remember it, as in the case presented by Grant ${ }^{7}$ in 1927. In the current work, patients on drugs affecting hemostasis had CSDH without a history of head trauma more frequently (61\%) than those with such history (39\%) and the difference was statistically significant. Aspegren et al. ${ }^{1)}$ made similar observations finding that patients who are receiving anticoagulants or antiplatelet therapy are in increased risk of CSDH formation even without trauma.

One explanation for hematoma enlargement is related to recurrent bleeding from neocapillaries in the hematoma capsule, which Putnam and Cushing first proposed. Gardner's conception related to differences in oncotic pressure but Weir questioned this idea in $1980^{23)}$. Dynamics of CSDH formation in patients on drugs affecting hemostasis may be higher when combined with Putnam's hypothesis of fragile neocapillaries.

Similar to what was observed in 2300 patients with $\mathrm{CSDH}^{18)}$ in Sambasivan's publication, we observed mainly unilateral hematomas. In our two groups, left-sided hematomas were more common, in accordance with other reports ${ }^{6,14)}$. One proposed explanation for the laterality of CSDH is based on skull asymmetry and was presented by Kim et al. ${ }^{14)}$. The possibility of new focal deficit is higher when the hematoma is located on the side of the dominant hemisphere with the risk of speech area compression, which may also explain why left-sided hematomas are more commonly diagnosed.

On admission, CT scan revealed hematomas of comparatively smaller thickness in the group on drugs affecting hemostasis. This difference was statistically significant between the groups. From patients on drugs affecting hemostasis only those who were on OAD had statistically significant difference in hematoma thickness as compared to those without bleeding disorders. on drugs affecting hemostasis. We have not found a clear explanation of this phenomenon in the available literature. Autoregulation mechanisms under CSDH seem to be intact and are responsible for cerebral perfusion changes ${ }^{20)}$. We infer that bleeding disorders may result in more rapid enlargement of hematoma, which gives less time for upregulating autoregulation and leads to an earlier appearance of symptoms and signs. In some cases of small hematoma, neurological deficit can be explained by a seizure accompanied by changes in electroencephalography. Hamasaki et al. ${ }^{9)}$ found a seizure as a symptom in $6 \%$ of patients with $\mathrm{CSDH}$.

Options for reversing bleeding disorders in patients on OADs include discontinuing medication ${ }^{24)}$, administering vitamin K, FFP or PCC. When the time from the last OAD intake was shorter than 3 hours, up to 100 grams of activated carbon can be used to reduce gastrointestinal absorption. For a long-term compensation of bleeding disorders, 5-10 mg of vitamin $\mathrm{K}$ in 30 minute infusion is prescribed. As mentioned above, FFP and PCC can be used for reversion of medication. PCC is preferable to FFP due to such side effects of FFP as Transfusion-Related Acute Lung Injury and a long preparation time $e^{26)}$.

In the case of bleeding in patients on high-dose ASA or newer antiplatelet drugs, such as Clopidogrelum in stable patients, medication discontinuation may be sufficient; in other cases, however, platelet concentration transfusion might be 
necessary. As suggested by papers about reversal of antiplatelet medication in terms of intracerebral hemorrhage in patients who are on ASA, 5 units of platelets is enough to reverse its effect. In those who are on any of thienopyridine medication, up to 10 units may be required to reverse the effect of medication. In some cases Desmopressin transfusion may also be helpful ${ }^{4)}$.

Reversal of LMWH is achieved after 12 hours from the last administration. In emergency cases, $1 \mathrm{mg}$ of Protamine Sulfate reverses 100 units of unfractionated heparin or $1 \mathrm{mg}$ of $\mathrm{LMWH}^{26)}$.

Symptomatic hematomas should be treated with hematoma evacuation. Nevertheless, in stable patients with minimal symptoms and without being on drugs affecting hemostasis, medical therapy with tranexamic acid ${ }^{12)}$ can be taken into consideration, as described by Kageyama and colleagues. However, this kind of pharmacotherapy in in patients on drugs affecting hemostasis is contraindicated because of an increased risk of embolic complications.

In this patient population, neurological deficit increased in two cases during bleeding disorder reversal; however, these patients improved after a surgery and were discharged without neurological deficit. Reversal of anticoagulation was not related to any thromboembolic complications in the group on drugs affecting hemostasis as a whole.

The standard procedure in our department in case of $\mathrm{CSDH}$ is frontal and parietal burr hole trephination with temporary external drainage of the subdural space. Even though some authors state that one burr hole is sufficient to achieve a good surgical result ${ }^{10)}$. Bleeding disorders did not influence the decision about the extent of the procedure in the analyzed series : the percentage of one or two burr holes and craniotomy was similar between groups, as was the risk of recurrent hematoma (9\% in group without bleeding disorders vs. 7.5\% in the group on drugs affecting hemostasis). In general, the risk of recurrent hematoma ranges from $1 \%$ to $17 \%{ }^{16,18)}$. According to Wada et al. ${ }^{22)}$ preoperative oral antiplatelet agents may increase the risk of hematoma recurrence up to $32 \%$. We did not find the statistical difference between the APA subgroup and the group without bleeding disorders in terms of hematoma recurrence.

In patients with higher risk of thromboembolic complications, enoxaparin at $40 \mathrm{mg} /$ day was administered a few hours after removal of the external drainage. Contrary to what was presented in a paper Tahsim-Oglou et al. ${ }^{21)}$, analysis of our series showed that postoperative prophylaxis with LMWH is not associated with a higher rate of hematoma recurrence. In patients who require discontinuation of treatment with antiplatelet therapy, ASA therapy should be continued in perioperative period and the previous treatment should be restored as soon as possible ${ }^{8}$.

Proper intravenous fluid administration in postoperative care may improve the outcome and diminish the rate of recurrent hematoma ${ }^{11}$. We found no differences between the groups. In addition, results of operative treatment according to the GOS and recurrence rate in long-term follow-up were similar in both groups.

\section{CONCLUSION}

Patients with CSDH who are on drugs affecting hemostasis are less often aware of a head trauma. Short-term anticoagulation reversal did not increase thromboembolic complications and the results of treatment. Results of treatment in both groups were similar and drugs affecting hemostasis does not influence the rate of $\mathrm{CSDH}$ recurrence.

\section{References}

1. Aspegren $\mathrm{OP}$, Åstrand R, Lundgren MI, Romner B : Anticoagulation therapy a risk factor for the development of chronic subdural hematoma. Clin Neurol Neurosurg 115 : 981-984, 2013

2. Baechli $H$, Nordmann A, Bucher HC, Gratzl 0 : Demographics and prevalent risk factors of chronic subdural haematoma : results of a large single-center cohort study. Neurosurg Rev 27 : 263-266, 2004

3. Bret P, Lecuire J, Lapras C, Deruty R, Desgeorges M, Prudhon JL : Subdural hematoma and anticoagulant therapy. Neurochirurgie 22 : 603620, 1976

4. Campbell PG, Sen A, Yadla S, Jabbour P, Jallo J : Emergency reversal of antiplatelet agents in patients presenting with an intracranial hemorrhage : a clinical review. World Neurosurg $74:$ 279-285, 2010

5. Forster MT, Mathé AK, Senft C, Scharrer I, Seifert V, Gerlach R : The influence of preoperative anticoagulation on outcome and quality of life after surgical treatment of chronic subdural hematoma. J Clin Neurosci $17: 975-979,2010$

6. Gelabert-González M, Iglesias-Pais M, García-Allut A, Martínez-Rumbo $R$ : Chronic subdural haematoma : surgical treatment and outcome in 1000 cases. Clin Neurol Neurosurg 107 : 223-229, 2005

7. Grant FC : Chronic subdural haematoma. Ann Surg 86 : 485-493, 1927 
8. Grines CL, Bonow RO, Casey DE Jr, Gardner TJ, Lockhart PB, Moliterno DJ, et al. : Prevention of premature discontinuation of dual antiplatelet therapy in patients with coronary artery stents : a science advisory from the american heart association, american college of cardiology, society for cardiovascular angiography and interventions, american college of surgeons, and american dental association, with representation from the american college of physicians. Circulation 115 : 813-818, 2007

9. Hamasaki T, Yamada K, Kuratsu J : Seizures as a presenting symptom in neurosurgical patients : a retrospective single-institution analysis. Clin Neurol Neurosurg 115 : 2336-2340, 2013

10. Han HJ, Park CW, Kim EY, Yoo CJ, Kim YB, Kim WK : One vs. two burr hole craniostomy in surgical treatment of chronic subdural hematoma. J Korean Neurosurg Soc 46 : 87-92, 2009

11. Janowski $\mathrm{M}$, Kunert $\mathrm{P}$ : Intravenous fluid administration may improve post-operative course of patients with chronic subdural hematoma : a retrospective study. PLoS One 7 : e35634, 2012

12. Kageyama H, Toyooka T, Tsuzuki N, Oka K : Nonsurgical treatment of chronic subdural hematoma with tranexamic acid. J Neurosurg 119 : 332-337, 2013

13. Kanat A, Kayaci S, Yazar U, Kazdal H, Terzi Y : Chronic subdural hematoma in adults : why does it occur more often in males than females? Influence of patient's sexual gender on occurrence. J Neurosurg Sci 54 : 99-103, 2010

14. Kim BG, Lee KS, Shim JJ, Yoon SM, Doh JW, Bae HG : What determines the laterality of the chronic subdural hematoma? J Korean Neurosurg Soc 47 : 424-427, 2010

15. Kunert $P$, Koszewski $W$ : At the end of the 20th century those patients accounted only for $1.2 \%$ of all patients with CSDH in our Department.

16. Lindvall P, Koskinen LO : Anticoagulants and antiplatelet agents and the risk of development and recurrence of chronic subdural haematomas. J
Clin Neurosci 16 : 1287-1290, 2009

17. Miranda LB, Braxton E, Hobbs J, Quigley MR : Chronic subdural hematoma in the elderly : not a benign disease. J Neurosurg 114 : 72-76, 2011

18. Sambasivan $M:$ An overview of chronic subdural hematoma : experience with 2300 cases. Surg Neurol 47 : 418-422, 1997

19. Sim YW, Min KS, Lee MS, Kim YG, Kim DH : Recent changes in risk factors of chronic subdural hematoma. J Korean Neurosurg Soc 52 : 234-239, 2012

20. Slotty PJ, Kamp MA, Steiger SH, Cornelius JF, Macht S, Stummer W, et al. : Cerebral perfusion changes in chronic subdural hematoma. J Neurotrauma $30: 347-351,2013$

21. Tahsim-Oglou Y, Beseoglu K, Hänggi D, Stummer W, Steiger HJ : Factors predicting recurrence of chronic subdural haematoma : the influence of intraoperative irrigation and low-molecular-weight heparin thromboprophylaxis. Acta Neurochir (Wien) 154 : 1063-1067; discussion 1068, 2012

22. Wada M, Yamakami I, Higuchi Y, Tanaka M, Suda S, Ono J, et al. : Influence of antiplatelet therapy on postoperative recurrence of chronic subdural hematoma : a multicenter retrospective study in 719 patients. Clin Neurol Neurosurg $120:$ 49-54, 2014

23. Weir B : Oncotic pressure of subdural fluids. J Neurosurg 53 : 512-515, 1980

24. White RH, McKittrick T, Hutchinson R, Twitchell J : Temporary discontinuation of warfarin therapy : changes in the international normalized ratio. Ann Intern Med 122 : 40-42, 1995

25. Yamashima T, Friede RL : Why do bridging veins rupture into the virtual subdural space? J Neurol Neurosurg Psychiatry 47 : 121-127, 1984

26. Yates $S$, Sarode $R$ : Reversal of anticoagulant effects in patients with intracerebral hemorrhage. Curr Neurol Neurosci Rep 15 : 504, 2015 